\title{
MORPHOLOGICAL ABNORMALITIES IN 2-YEAR-OLD CHILDREN BORN AFTER IVF/ICSI WITH PREIMPLANTATION GENETIC SCREENING (PGS)
}

F. Beukers ${ }^{1}$, M. van der Heide ${ }^{1}$, K. Middelburg ${ }^{2}$, J.M. Cobben ${ }^{3}$, S. Mastenbroek ${ }^{4}$, R. Breur ${ }^{5}$, J.H. van der Lee $^{6}$, M. Hadders-Algra ${ }^{2}$, A.F. Bos ${ }^{7}$, J.H. Kok ${ }^{1}$

${ }^{I}$ Department of Neonatology, Academic Medical Center Amsterdam, Amsterdam, ${ }^{2}$ Department of Paediatrics, Division of Developmental Neurology, University Medical Center Groningen, Groningen, ${ }^{3}$ Department of Paediatrics, Clinical Genetics, ${ }^{4}$ Center for Reproductive Medicine, Academic Medical Center Amsterdam, Amsterdam, ${ }^{5}$ Public Health Service Zaanstreek-Waterland, Purmerend, ${ }^{6}$ Department of Pediatric Clinical Epidemiology, Academic Medical Center Amsterdam, Amsterdam, ${ }^{7}$ Department of Paediatrics, Division of Neonatology, University Medical Center Groningen, Groningen, The Netherlands

Background and aims: Increased risk for chromosomal abnormalities in embryos is considered as a cause of low pregnancy rate with advanced maternal age. PGS has been suggested to improve pregnancy outcome. Since PGS includes extensive embryo manipulation, PGS could have an effect on morphological outcome in children. A follow-up study of a RCT comparing IVF/ICSI with PGS (PGS+) and without (PGS-) was performed to evaluate morphological abnormalities of live born children at 2 years of age.

Methods: 408 women were included in a multicenter RCT on the efficacy of PGS. There were 120 women with $\geq 1$ live births, 119 mothers with 142 children alive (57 PGS+; 85 PGS-) were available for follow-up. A reference group of 67 children conceived naturally was formed. At 2 years of age a body-surface examination and anthropometry was performed. Morphological abnormalities were classified as major abnormalities, minor anomalies or common variants according to Merks (Am J Med Genet 2003;123A:211230).

Results: No significant association was found between PGS and any specific minor anomaly or major abnormality. The proportions [95\% CI] of children with $\geq 1$ minor anomalies and $\geq 1$ major abnormalities for PGS+ versus PGS- were $64 \%$ [50 -76] vs $67 \%$ [55 - 77] and $28 \%$ [18 - 42] vs $35 \%$ [25 - 46], respectively. No significant differences were found between the randomized and reference groups.

Conclusions: This study found no differences in morphological abnormalities between children conceived after IVF/ICSI with PGS and children conceived after regular treatment. 